\title{
Fuchs' Problem When Torsion-Free Abelian Rank-One Groups are Slender
}

\author{
PAVLOS TZERMIAS
}

\begin{abstract}
We combine Baer's classification in [Duke Math. J. 3 (1937), 68-122] of torsion-free abelian groups of rank one together with elementary properties of $p$-adic numbers to give a new solution to research problem 26 posed by Fuchs in his book on abelian groups in 1958.
\end{abstract}

Every group considered in this paper is abelian with its group law written additively. The Baer-Specker group $\Pi=\mathbb{Z}^{\aleph_{0}}$ is the direct product of countably many copies of $\mathbb{Z}$. Its extensive study in the literature has given rise to a wealth of interesting problems and a number of unexpected connections with diverse mathematical areas such as homology theories in algebraic topology, infinitary logic and various aspects of set theory. We refer the reader to [2], [3], [4], [6] and [11] for good accounts of some of these connections.

For a positive integer $n$, let $e_{n}$ denote the element of $\Pi$ whose $n$-th coordinate equals 1 and all its other coordinates equal 0. Following Łoś, a torsion-free group $G$ is called slender if for every homomorphism $\phi: \Pi \rightarrow G$ we have $\phi\left(e_{n}\right)=0$, for all but finitely many $n$. Specker ([10]) showed that $\mathbb{Z}$ is slender. Research problem 26 in Fuchs' book (page 184 in [5]) reads as follows:

Problem. Find all slender groups of rank one.

A partial answer to the problem was given by Łoś (see Theorem 47.3 in [5]). The problem was fully solved by Sąsiada ([9]) who in fact showed that:

Theorem 1. A torsion-free group of cardinality less than $2^{\aleph_{0}}$ is slender if and only if it has no non-trivial divisible subgroups.

2000 Mathematics Subject Classification. Primary 20K15; Secondary 20K25.

Key words and phrases. Rank-one groups, slender groups, Baer-Specker group. 
Sąsiada's result is superseded by Nunke's characterization of all torsion-free groups which are slender (see [7] and [8]).

Theorem 2. A torsion-free group is slender if and only if none of its subgroups is isomorphic to $\Pi, \mathbb{Q}$ or the p-adic integers $\mathbb{Z}_{p}$ for some prime $p$.

The purpose of this short note is to give a new solution to the original problem of Fuchs. We claim no novelty for the general $p$-adic flavor of our arguments which is not uncommon in inquiries of this type but, as far as we can tell, a solution like ours has not appeared in the literature before. Let us first briefly recall Baer's classification ([1]) of torsion-free groups of rank one (see [5] for details).

Consider a torsion-free group $G$ and a non-zero element $g \in G$. For a prime number $p$, define the height $H_{p}(g)$ of $g$ at $p$ to be the maximum integer $k \in \mathbb{N}$ such that the equation $p^{k} x=g$ is solvable in $G$ (if no maximum $k$ exists, we set $H_{p}(g)=\infty$ ). The height $H(g)$ of $g$ is the infinite tuple

$$
H(g)=\left(H_{p_{1}}(g), H_{p_{2}}(g), \ldots, H_{p_{n}}(g), \ldots\right),
$$

where $p_{1}, p_{2}, \ldots, p_{n}, \ldots$ is the increasing sequence of prime numbers.

Consider the set $H$ of infinite tuples $\left(k_{1}, k_{2}, \ldots, k_{n}, \ldots\right)$, where $k_{n} \in \mathbb{N} \cup\{\infty\}$ for all $n$. Define a partial order $\leq$ on $H$ as follows:

$$
\left(k_{1}, k_{2}, \ldots, k_{n}, \ldots\right) \leq\left(m_{1}, m_{2}, \ldots, m_{n}, \ldots\right)
$$

if $k_{n} \leq m_{n}$ for all $n$ (where it is understood that $s \leq \infty$ for all $s \in \mathbb{N} \cup\{\infty\})$.

We also define an equivalence relation $\sim$ on $H$ as follows:

$$
\left(k_{1}, k_{2}, \ldots, k_{n}, \ldots\right) \sim\left(m_{1}, m_{2}, \ldots, m_{n}, \ldots\right)
$$

provided that $k_{n}=m_{n}$ for all but finitely many $n$ and that $k_{n} \neq m_{n}$ can happen only if neither $k_{n}$ nor $m_{n}$ equals $\infty$. An equivalence class of $H$ under $\sim$ is called a type and the set of all types is denoted by $T$. It is easy to see that the partial order $\leq$ on $H$ defined above induces a partial order (also denoted by $\leq$ ) on the set of types $T$.

Now suppose that $G$ is of rank one and let $g, g^{\prime}$ be any two nonzero elements of $G$. It is not difficult to show that $H(g) \sim H\left(g^{\prime}\right)$. Therefore, all non-zero elements of $G$ are of the same type, which we call the type of $G$ and denote by $T(G)$. Every torsion-free group of rank one is isomorphic to a subgroup of $\mathbb{Q}$ and Baer showed in [1] that the set of isomorphism classes of torsion-free groups of rank one is parametrized by $T$ via the bijective correspondence given by 
$G \mapsto T(G)$. In addition, a torsion-free group $G_{1}$ of rank one is isomorphic to a subgroup of a torsion-free group $G_{2}$ of rank one if and only if $T\left(G_{1}\right) \leq T\left(G_{2}\right)$.

We now give a new solution to the original problem of Fuchs:

Theorem 3. A torsion-free group of rank one is slender if and only if it is not isomorphic to $\mathbb{Q}$.

Proof. One direction is standard: Let $\Sigma$ denote the direct sum of countably many copies of $\mathbb{Z}$. Since $\mathbb{Q}$ is an injective $\mathbb{Z}$-module, the homomorphism $\Sigma \rightarrow \mathbb{Q}$ defined by

$$
\left(x_{1}, x_{2}, \ldots, x_{n}, \ldots\right) \mapsto \sum_{n=1}^{\infty} x_{n}
$$

can be lifted to a homomorphism $\phi: \Pi \rightarrow \mathbb{Q}$ such that $\phi\left(e_{n}\right) \neq 0$, for all $n$. Therefore, $\mathbb{Q}$ is not slender.

To prove the converse, let $G$ be a torsion-free group of rank one which is not isomorphic to $\mathbb{Q}$. From the discussion of types given before, it follows that $T(G)$ can be represented by an infinite tuple $\left(k_{1}, k_{2}, \ldots, k_{n}, \ldots\right)$, where $k_{n} \neq \infty$ for at least one $n \in \mathbb{N}$. It is easy to see that this tuple is equivalent to $\left(k_{1}, k_{2}, \ldots, k_{n-1}, 0, k_{n+1}, \ldots\right)$ and that

$$
\left(k_{1}, k_{2}, \ldots, k_{n-1}, 0, k_{n+1}, \ldots\right) \leq(\infty, \infty, \ldots, \infty, 0, \infty, \ldots) .
$$

As the latter tuple represents the type of the group $\mathbb{Z}_{(p)}$ of rational numbers with denominators not divisible by the prime $p=p_{n}$, we may assume that $G$ is a subgroup of $\mathbb{Z}_{(p)}$. Since subgroups of slender groups are obviously slender, it suffices to show that $\mathbb{Z}_{(p)}$ is slender.

Let $i: \mathbb{Z}_{(p)} \rightarrow \mathbb{Z}_{p}$ denote the natural embedding of $\mathbb{Z}_{(p)}$ in the ring of $p$-adic integers. We will use the well-known fact that for $y \in \mathbb{Z}_{(p)}$ the $p$-adic expansion of $i(y)$ is ultimately periodic, i.e. when we write

$$
i(y)=\sum_{j=0}^{\infty} a_{j} p^{j}
$$

then there exist positive integers $N$ and $r$ such that $a_{j+r}=a_{j}$ for all $j \geq N$. Now let $\phi: \Pi \rightarrow \mathbb{Z}_{(p)}$ be a homomorphism such that $\phi\left(e_{n}\right) \neq 0$ for infinitely many $n$. Without loss of generality, we may assume that $\phi\left(e_{n}\right) \neq 0$ for all $n$. We can therefore find, for each $n$, an integer $b_{n}$ such that $\phi\left(b_{n} e_{n}\right)$ is a positive integer. Let $y_{n}=\phi\left(b_{n} e_{n}\right)$. Then $i\left(y_{n}\right)$ has a finite non-zero $p$-adic expansion for all $n$. Now 
consider $x=\left(p^{c_{1}} b_{1}, p^{c_{2}} b_{2}, \ldots\right) \in \Pi$, where the sequence of natural numbers $c_{1}, c_{2}, \ldots$ has been chosen to satisfy

$$
p^{c_{n+1}}>p^{n}\left(p^{c_{n}} y_{n}+p^{c_{n-1}} y_{n-1}+\ldots+p^{c_{1}} y_{1}\right)
$$

for all $n$. Let $y=\phi(x)$. Note that for all $n$ we get

$y=p^{c_{1}} y_{1}+p^{c_{2}} y_{2}+\ldots+p^{c_{n}} y_{n}+\phi\left(0,0, \ldots, 0, p^{c_{n+1}} b_{n+1}, p^{c_{n+2}} b_{n+2}, \ldots\right)$.

We clearly have $c_{n+1} \leq c_{n+2} \leq \ldots$, so

$$
\left(0,0, \ldots, p^{c_{n+1}} b_{n+1}, p^{c_{n+2}} b_{n+2}, \ldots\right)
$$

is divisible by $p^{c_{n+1}}$ in $\Pi$. Hence,

$$
y \equiv p^{c_{1}} y_{1}+p^{c_{2}} y_{2}+\ldots+p^{c_{n}} y_{n} \quad\left(\bmod p^{c_{n+1}}\right) .
$$

for all $n$. By our assumption on $c_{1}, c_{2}, \ldots$, we see that the $p$-adic expansion of $i(y)$ is obtained by writing down the non-zero $p$-adic expansion of $p^{c_{1}} i\left(y_{1}\right)$, followed by at least 1 zero digit, followed by the non-zero $p$-adic expansion of $p^{c_{2}} i\left(y_{2}\right)$, followed by at least 2 zero digits, etc. Hence, the $p$-adic expansion of $i(y)$ contains arbitrarily large blocks of zero digits followed by non-zero blocks of digits and this contradicts the fact that the $p$-adic expansion of $i(y)$ is ultimately periodic.

Acknowledgement. I thank Yasuyuki Kachi for asking me a question which, unbeknownst to me at the time, was equivalent to the slenderness of $\mathbb{Z}$ and provided the motivation for this work.

\section{REFERENCES}

[1] R. Baer, Abelian groups without elements of finite order, Duke Math. J. 3 (1937), 68-122.

[2] A. Blass and J. Irwin, Free subgroups of the Baer-Specker group, Comm. in Algebra 29 (2001), no. 12, 5769-5794.

[3] E. Coleman, The Baer-Specker group, Ir. Math. Soc. Bull. 40 (1998), 9-23.

[4] P. Eklof and A. Mekler, Almost free modules. Set-theoretic methods, Revised Edition, North Holland Publishing Co., Amsterdam, 2002.

[5] L. Fuchs, Abelian groups, Publishing House of the Hungarian Academy of Sciences, Budapest, 1958

[6] W. Massey, How to give an exposition of the Čech-Alexander-Spanier type homology theory, Amer. Math. Monthly 85 (1978), no. 2, 75-83.

[7] R. Nunke, Slender groups, Bull. Amer. Math. Soc. 67 (1961), 274-275.

[8] R. Nunke, Slender groups, Acta Sci. Math. (Szeged) 23 (1962), 67-73.

[9] E. Sąsiada, Proof that every countable and reduced torsion-free Abelian group is slender, Bull. de l'Acad. Polonaise des Sciences, Série des Sciences Mathématiques, Astronomiques et Physiques 7 (1959), 143-144. 
[10] E. Specker, Additive Gruppen von Folgen ganzer Zahlen, Portugaliae Mathematica 9 (1950), 131-140.

[11] E. Zeeman, On direct sums of free cycles, J. London Math. Soc. 30 (1955), 195-212.

Pavlos Tzermias,

Department of Mathematics,

University of Tennessee,

104 Aconda Court,

1534 Cumberland Avenue,

Knoxville, TN 37996-0612, USA

tzermias@math.utk.edu

Received on 15 December 2008. 